\title{
Numerical Simulation of Asymmetrically Altered Growth as Initiation Mechanism of Scoliosis
}

\author{
A. van der Platts, ${ }^{1}$ A. G. Veldhuizen, ${ }^{2}$ and G. J. Verkerke ${ }^{1,3}$ \\ ${ }^{1}$ Department of Biomedical Engineering, University Medical Center Groningen, University of Groningen, Antonius Deusinglaan 1, \\ 9713 AV Groningen, The Netherlands; ${ }^{2}$ Department of Orthopedic Surgery, University Medical Center Groningen, University \\ of Groningen, Groningen, The Netherlands; and ${ }^{3}$ Dept of Biomechanical Engineering, University of Twente, Enschede, \\ The Netherlands
}

(Received 12 December 2005; accepted 8 January 2007; published online 6 April 2007)

\begin{abstract}
The causes of idiopathic scoliosis are still uncertain; buckling is mentioned often, but never proven. The authors hypothesize another option: unilateral postponement of growth of MM Rotatores or of ligamentum flavum and intertransverse ligament. In this paper, both buckling and the two new theories of scoliotic initiation are studied using a new finite element model that simulates the mechanical behavior of the human spine. This model was validated by the stiffness data of Panjabi et al. (J. Biomech. 9:185-192, 1976). After a small correction of the prestrain of some ligaments and the MM Rotatores the model appeared to be valid. The postponement in growth was translated in the numerical model in an asymmetrical stiffness. The spine was loaded axially and the resulting deformation was analyzed for the presence of the coupling of lateral deviation and axial rotation that is characteristic for scoliosis. Only unilateral postponement of growth of ligamentum flavum and intertransverse ligament appeared to initiate scoliosis. Buckling did not initiate scoliosis.
\end{abstract}

Keywords-Scoliosis, Biomechanics, Idiopathic, Finite element method, Buckling, Numerical simulation, Growth postponement.

\section{INTRODUCTION}

Scoliosis, a three-dimensional (3D) deformity of the human spinal column, is characterized by a lateral deviation of the spine, accompanied by an axial rotation of the vertebrae. This axial rotation is such that the spinous processes of the vertebrae rotate toward the concave side of the lateral curvature. Maximum progression in scoliosis has shown to be during growth.

About $80 \%$ of all cases of scoliosis are idiopathic. Despite many research, the cause of scoliosis is still unknown. ${ }^{15,18}$ White $^{37}$ postulated the theory that a

Address correspondence to G. J. Verkerke, Department of Biomedical Engineering, University Medical Center Groningen, University of Groningen, Antonius Deusinglaan 1, 9713 AV Groningen, The Netherlands. Electronic mail: g.j.verkerke@med.umcg.nl scoliotic spine most probably results from a slight disturbance in the critical balance between lateral deviation and axial rotation in a motion segment. This theory is widely accepted for explaining scoliosis. One of the most presumed causes of disturbance, mentioned in the literature is buckling of the vertebral column. The idea behind this theory is that the buckling phenomenon causes an initial lateral curvature with axial rotation, which progresses by growth. Dickson, ${ }^{6,7}$ Millner and Dickson, ${ }^{19}$ and Smith and Dickson $^{30}$ mentioned buckling as an initiation of idiopathic scoliosis by using the linear Euler buckling theory. Gross et al. ${ }^{12}$ came in their analysis of literature to the same conclusion: initiation of scoliosis has to be found in a buckling theory. Recently, Goto et al. ${ }^{11}$ studied buckling of the spine and found that buckling could cause scoliosis. They used a very detailed finite element model for this study. However, it was a linear model that did not include ligaments, articulations, and muscles. As they mentioned actually 'a more realistic model that includes these structures is necessary'.

Beguiristain et al., ${ }^{3}$ using two-month-old pigs, performed selective epiphysiodesis of the neurocentral cartilage with the aid of cancellous screw insertion achieving effective compression. In other animals treated at ages when the neurocentral cartilage is not active rotational deformity did not occur.

Alexander et al. ${ }^{1}$ produce scoliosis in a rabbit using dorsal rhiztomy. The scoliosis produced resembles a clinical paralytic type of scoliosis, not an idiopathic type.

Langenskiöld and Michelsson ${ }^{14}$ performed unilateral resection of the posterior ends of the sixth to eleventh ribs in young rabbits including the costal parts of both costo-vertebral joints. They found that progressive scoliosis can be provoked. Sevastikoglou ${ }^{29}$ performed a comparable study with similar results. 
An attempt to produce scoliosis in young baboons by excision of the head of ribs failed. ${ }^{25}$ Other investigators have failed to produce scoliosis in primates by similar and other technique that had successfully produced scoliosis in quadruped animals.

Stokes and Gardner-Morse ${ }^{32}$ analyzed, using a finite element model, the effect of tethering by the posterior soft tissues, as a mechanism of causing the typical scoliotic curvature. They found the coupled interaction between lateral deviation and axial rotation, although very small. A more thorough investigation on the actual structures causing this interaction is, however, necessary.

Others propose a neuromuscular cause of idiopathic scoliosis resulting in asymmetric muscle properties that disturb the force balance and produce a scoliotic curve. ${ }^{13,36}$ This was clinically justified by the finding that a left-right asymmetry in spinal muscles appeared to correlate with scoliosis progression. ${ }^{4}$

In our study three mechanical and growth-related possible causes of scoliosis are analyzed. First, buckling is studied using a more realistic non-linear model than that of Goto et al. ${ }^{11}$ Next, the theory of Veldhuizen et al., ${ }^{36}$ and Herman et al., ${ }^{13}$ assuming unilaterally postponement in growth of muscles, resulting in asymmetric muscle properties that could lead to asymmetric behavior of the spine is studied. Finally the authors postulate an alternative cause of scoliosis, derived from the theory of Veldhuizen et al. ${ }^{36}$ and Herman et al. ${ }^{13}$ and following the theory of Stokes and GardnerMorse $^{32}$ : unilaterally postponement in growth of ligaments of the spine resulting in asymmetric ligament properties leading to asymmetric behavior of the spine. Especially two ligaments, L Flavum and L Intratransversalis have such an orientation that they could induce scoliosis when growth is postponed unilaterally.

To study buckling and unilateral postponement in growth a numerical simulation model of the spine is appropriate, since numerical models make it possible to analyze the mechanical behavior of a complex and highly non-linear structure as the human vertebral column. In the past many of these models have been developed, varying from very detailed micro models ${ }^{10}$ for close studies of the mechanical behavior on motion segment level, to simple macro models ${ }^{2,5,24,33,34}$ for studies of the mechanical behavior of the entire spinal column. Characteristic of a micro model is that very detailed studies can be done, but an overview is not possible. Using a macro model an overview becomes possible, but the influence of different structures of the spine cannot be included. For this matter, Gilbertson et al. ${ }^{9}$ developed a combined model, consisting of a thoracic macro model and a lumbar micro model. Goto et al. ${ }^{11}$ used a detailed finite element model of the complete spine for a buckling study.
For our study of the initiation of idiopathic scoliosis, an overview of the entire spinal column is necessary, but also all the important structures that determine the highly non-linear biomechanical behavior of the spine have to be taken into account. So a new finite element model is developed that combines macro and micro models, so detailed structures are present while still the entire spinal column can be studied in three dimensions.

Including all spinal muscles will result in a more realistic model, we agree upon that. However, a large amount of muscle structures is involved in the stability of the spine and their orientation is very complex. It is only recently that the 3-D anatomy of the spinal muscles is accurately and fully determined in terms of the coordinates of origin and insertio. ${ }^{23}$ The large amount of muscles and complexity of their orientation makes it very difficult to include muscles in numerical models or to combine their action into a single follower load. This is the reason that in all numerical models of the spine, mentioned above, muscles are lacking. We have decided to follow their strategy and to develop a numerical model with only three muscle groups.

This paper describes a study on the initiation of idiopathic scoliosis by (1) buckling, (2) by unilaterally postponed growth of muscles, and (3) by unilaterally postponed growth of ligaments using a non-linear finite element numerical model of the spine.

\section{MATERIALS AND METHODS}

\section{Materials}

A finite element model is developed for this study to analyze the initiation of idiopathic scoliosis. The model is implemented in a finite element (FE) program (ANSYS version 5.3, Ansys Inc, Canonsburg PA 15317, USA).

On motion segment level the model is composed of the vertebrae, the intervertebral disc (IVD), the facet joints (FJ) and the spinal ligaments. Also an important group of spinal musculature, the MM Rotatores (MMR), is included. The vertebra is build up by its main structures: the transverse processes (TP), spinous processes (SP), articular processes (AP), laminae (LAM) and vertebral body (VB), all modeled by 3D elastic beam elements. These elements contain length, cross-sectional area, moments of inertia, thickness, and Young's and shear modulus. The location of the vertebral centra of the FE model are derived from X-ray photographs of human spines, the geometry of the vertebrae is based on Scoles et al. ${ }^{28}$ The facet joints are modeled using shell and contact elements. 
The vertebral material, mainly bone, is considered to be linear elastic and isotropic.

The material properties of bone are taken from Pitzen et al. ${ }^{22}$ and Evans ${ }^{8}$ and are: Young's modulus $E_{\text {bone }}=1.21 \times 10^{4} \mathrm{~N} / \mathrm{mm}^{2}$ and shear modulus $G_{\text {bo- }}$ $\mathrm{ne}=5.0 \times 10^{3} \mathrm{~N} / \mathrm{mm}^{2}$. The intervertebral disc is also modeled by a $3 \mathrm{D}$ elastic beam element. The values of intervertebral disc Young's and shear moduli are derived and averaged from Markoff, ${ }^{17}$ Schultz et al. ${ }^{27}$ and Panjabi et al. ${ }^{21}$ and have become: $E_{\mathrm{ivd}}=7 \mathrm{~N} /$ $\mathrm{mm}^{2}$ and $G_{\mathrm{ivd}}=2.6 \mathrm{~N} / \mathrm{mm}^{2}$, respectively. The viscoelastic properties of the intervertebral disc have not been accounted for, because only static behavior is analyzed.

In accordance with White and Panjabi ${ }^{38}$ seven spinal ligaments per motion segment are modeled: the intertransverse ligament (ITL), posterior longitudinal ligament (PLL), anterior longitudinal ligament (ALL), supra- and interspinous ligament (SSL and ISL), facet capsular ligament (FCL), and the ligamentum flavum (LF). All these ligaments are modeled by cable elements, which have only stiffness under tension, and zero stiffness under pressure. According to several authors $^{20,35}$ spinal ligaments are under prestrained conditions, so in this FE model an arbitrary initial strain of $0.5 \%$ is applied to the ligaments. Ligaments show a non-linear behavior. The stress-strain curve starts progressively, followed by a linear part and again a non-linear part. Since we assume prestress, we start in the linear part of the curve. Because the deformations remain limited, the last non-linear part of the graph will not be reached. A linear behavior of the ligaments is realistic within these boundaries. The Young's moduli and cross-sectional area's are listed in Table 1.

The MM Rotatores (MMR) are also modeled by cable elements. These muscles run from the transverse processes of the lower vertebra to the spinous process of the upper vertebra. Their main function is to stabilize the spinal column. Since we are analyzing static behavior, only the passive component of this musculature is taken into account. Relevant properties are

TABLE 1. Stiffness and geometric properties of the tensiononly elements used in ligament and muscle modeling ${ }^{27,33}$.

\begin{tabular}{lcc}
\hline $\begin{array}{l}\text { Ligament/ } \\
\text { muscle }\end{array}$ & $\begin{array}{r}\text { Young's modulus } \\
{\left[\mathrm{N} / \mathrm{mm}^{2}\right]}\end{array}$ & $\begin{array}{c}\text { Cross-sectional } \\
\text { area }\left[\mathrm{mm}^{2}\right]\end{array}$ \\
\hline ALL & 8 & 50 \\
PLL & 8 & 25 \\
FCL & 8 & 20 \\
ITL & 5 & 10 \\
LF & 24 & 30 \\
ISL & 5 & 26 \\
SSL & 5 & 26 \\
MMR & 25 & 10 \\
\hline
\end{tabular}

included in Table 1. Again a prestrain of $0.5 \%$ is applied to these elements.

Figure 1 shows the 3D FE model of a motion segment.

Using the previous mentioned modeled structures, a $3 \mathrm{D}$ model of the human thoracolumbar spine is modeled, consisting of 17 vertebrae, (see Fig. 2). The facet joints are differently orientated for the lumbar and thoracic region, as is described by Panjabi et al. ${ }^{21} \mathrm{In}$ the lumbar region the facet joints are placed $45^{\circ}$ out of the frontal plane, parallel to the longitudinal axis. In the thoracic region they are placed $20^{\circ}$ out of the frontal plane, and $30^{\circ}$ out of the transverse plane.

General boundary conditions in the analyses are a complete fixation at the S1-level and on the T1-level horizontal displacements and axial rotations are restricted, since in real life these displacements and rotations are also restricted by actions from the erector spinae muscles and the muscles of the back. By these boundary conditions the actions of the erector spinae muscles and the muscles of the back are indirectly included into the numerical model. The applied coordinate system is depicted in Fig. 2.

\section{Methods}

\section{Model Validation}

To validate the developed numerical model the results of the study of Panjabi et al. ${ }^{21}$ were used. A motion segment was loaded according to Panjabi's data. The resulting deformation was quantitatively compared with the deformation data of Panjabi.

To investigate buckling of the human spine as a possible initiation of idiopathic scoliosis, instability of the spine due to an axial downward force is studied. At this point of instability any small disturbance of the instable spine will cause a deformed shape, which we call the buckling mode. If it can be shown that the deformed shape includes a lateral deviation accompanied by an axial rotation of the vertebrae (spinous processes turn toward the concave side), like it occurs in scoliosis, it can be concluded that buckling of the spine is indeed a possible initiation of idiopathic scoliosis. In mathematical terms: axial rotation and lateral deviation must have the same sign.

The obtained (small) lateral deviation and axial rotation are believed to develop further into a serious scoliotic curvature due to growth.

In the finite element method, forces and displacements are related by the non-linearized tangent equation:

$$
\left(\left[K_{0}\right]+\left[K_{\mathrm{s}}\right]+\left[K_{\mathrm{t}}\right]\right) \cdot\{\dot{u}\}=\left\{F_{b}\right\} \cdot \dot{f}
$$



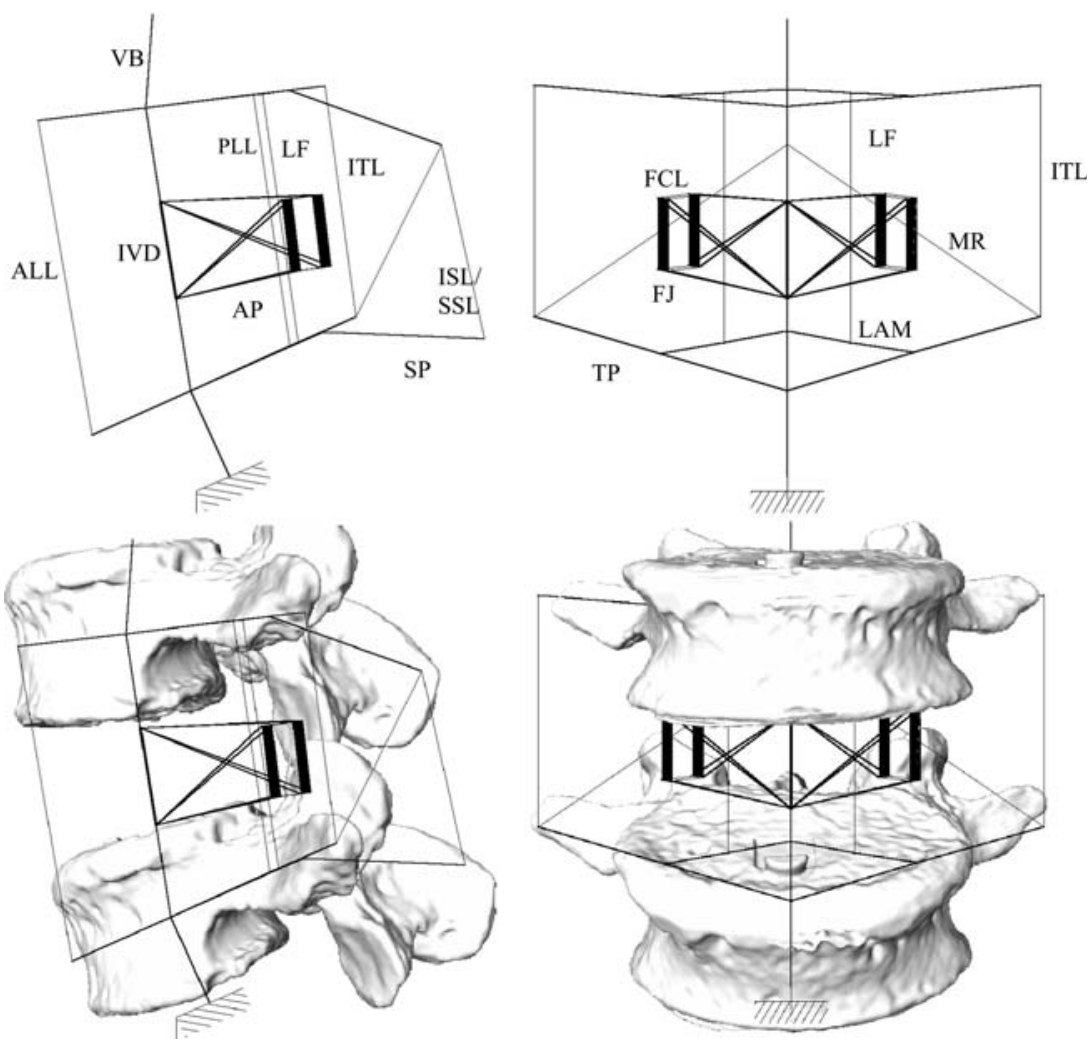

FIGURE 1. Side (left) and front (right) view of a motion segment with the modeled structures. For abbreviations, see text.

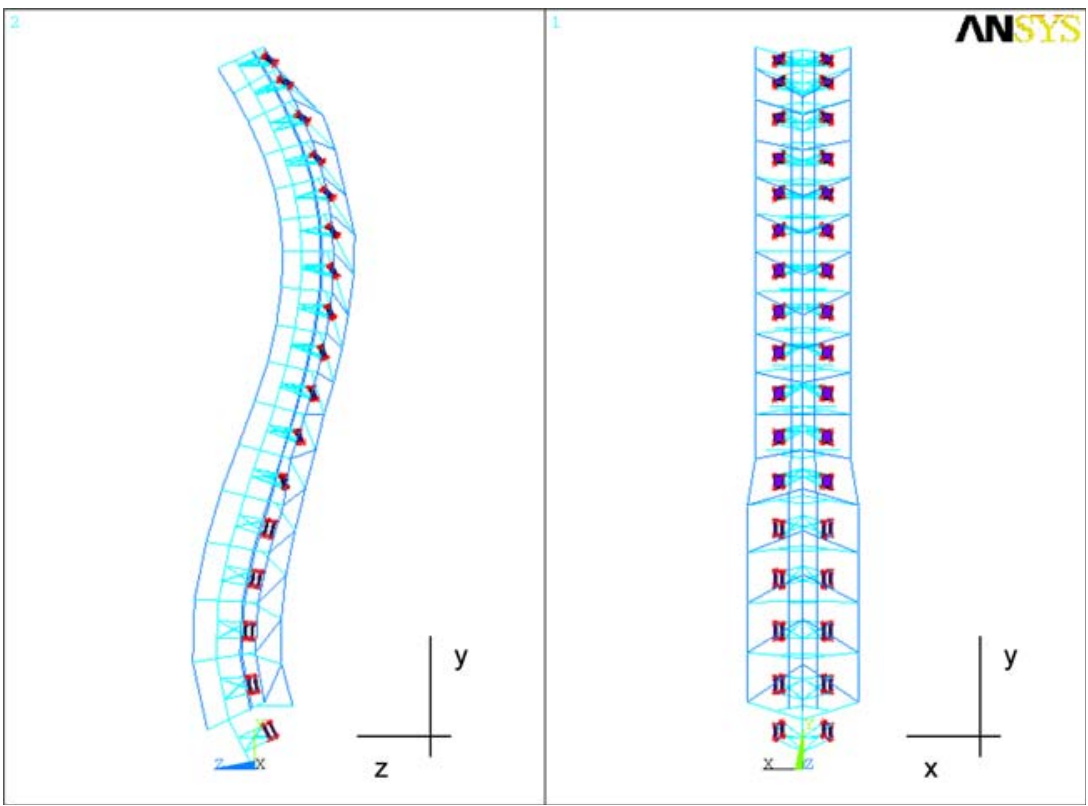

FIGURE 2. Side (left) and back (right) view of the 3D finite element model of the human thoracolumbar spine with a definition of the applied coordinate system.

for situations where $\dot{f}=0$ and $\dot{u} \neq 0$. Here $\left[K_{0}\right]$ is the stiffness matrix, $\left[K_{1}\right]$ the initial displacement matrix, $\left[K_{\mathrm{s}}\right]$ the initial stress matrix, $\{\dot{u}\}$ the first time-derivative of the displacement vector, $\left\{F_{\mathrm{b}}\right\}$ the force vector de- fined as $\partial F / \partial f$, and $\dot{f}$ the first time-derivative of the force. We had to use a non-linear strain-displacement relation because of large displacements (e.g., the Green-Lagrange strain). After linearization, the part 
of the stiffness matrix that depends on these displacements that are already present in the linearized state, is called the initial displacement matrix. The initial stress matrix depends on the stresses that are already present in the linearized state.

All 1500 nodes of the finite element model are included in the matrices. Solving these equations has been done by solving Eq. (2) iteratively using the Newton-Raphson iteration method.

$$
\operatorname{Det}\left(\left[K_{0}\right]+\left[K_{\mathrm{s}}\right]+\left[K_{l}\right]\right)=0
$$

\section{Hypothesis 1: Buckling}

The critical point of instability can be found by increasing the applied axial load until a maximum of potential energy at the equilibrium position in the force-displacement curve of the spine has been obtained. A disturbance at this point of instability has been initiated by a very small lateral eccentricity of the load.

\section{Hypotheses 2 and 3: Unilaterally Postponement in Growth of Muscles or Ligaments}

A unilaterally postponement in growth in ligaments or muscles will result in a larger stress in these structures. This can be simulated either by increasing the stiffness or increasing the prestress. The resulting effect, an increase in stress in the structures, is identical. So to investigate the influence of unilaterally postponed growth of muscles and ligaments the following changes in the numerical model were made:

1. The MMR are subjected to a unilaterally $20 \%$ increase in stiffness for the same reason to test hypothesis 2 .

2. The intertransverse ligament and the ligamentum flavum are subjected to a unilaterally $20 \%$ increase in stiffness to simulate the effect of postponed growth to test hypothesis 3 .

To initiate a lifelike response of the model, the top is loaded with a vertical pressure load of $500 \mathrm{~N}$, an average value for a person of normal stature in a relaxed, standing upright position. ${ }^{21}$ Part of this force is the weight of head and arms, part is muscle tone.

\section{RESULTS \\ Validation}

The validation study showed that the arbitrarily assumed prestrain of $0.5 \%$ had to be changed for some ligaments and the MMR. After adjusting the prestrain, differences in deformation between Panjabi and our model were less than $1 \%$. The resulting prestrain for all ligaments and muscles is presented in Table 2.

\section{Hypothesis 2: Buckling}

By increasing the axial force the point of mechanical instability was reached. Already the first buckling mode showed a scoliotic curve. The resulting deformation of the spinal column is shown in Fig. 3. However, the criteria of true scoliosis, axial rotation and lateral deviation with the same sign, are not met for the entire vertebral column, only for the lower thoracic and lumbar part (T8-L5).

\section{Hypotheses 2 and 3: Unilaterally Postponement in Growth of Muscles or Ligaments}

The results of increasing the stiffness of MMR and the intertransverse ligament and the ligamentum flavum unilaterally are shown in Figs. 4 and 5, respectively. In these figures, the deformation is given relative to a symmetrical deformation of the spine under an axial load of $500 \mathrm{~N}$ to have a better view on the effects of a unilateral increase of stiffness. Unilateral increase in stiffness of the ligaments results in scoliosis, since axial rotation and lateral deviation have the same sign. In contrast, unilateral increase in stiffness of the MMR does not result in scoliosis, since axial rotation and lateral deviation do not have the same sign.

\section{DISCUSSION}

The etiology of adolescent idiopathic scoliosis (AIS) remains an enigma. In the literature there are two opinions: one believes a deviating growth pattern is responsible for the condition (patients with AIS tend to be growing faster/taller) while the other opinion assumes that the growth pattern is normal, but its presence is necessary to allow the development of the scoliosis. The natural history of AIS involves an initial stage in which a small curve develops due to a small

TABLE 2. Prestrain of the ligaments and MMR after the validation study.

\begin{tabular}{lc}
\hline Ligament & Prestrain (\%) \\
\hline ALL & 2 \\
PLL & 0.5 \\
FCL & 1 \\
ITL & 0.5 \\
LF & 0.5 \\
ISL & 0.5 \\
SSL & 0.5 \\
MMR & 1 \\
\hline
\end{tabular}




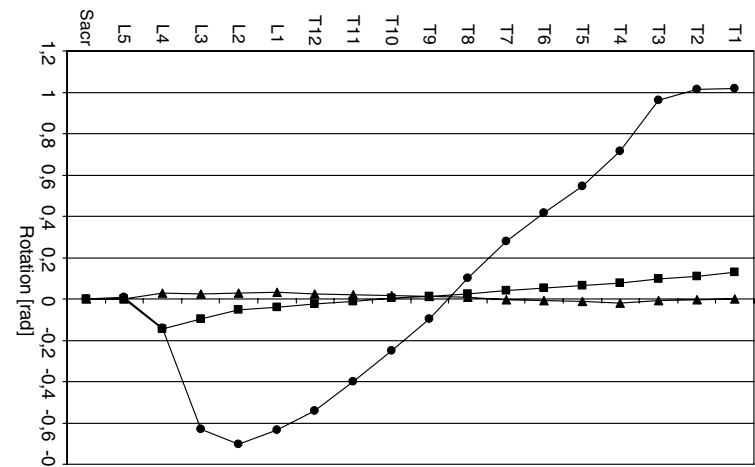

a

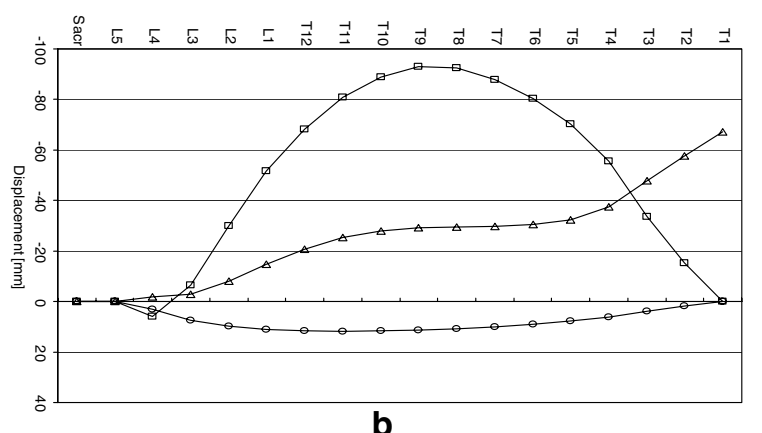

b
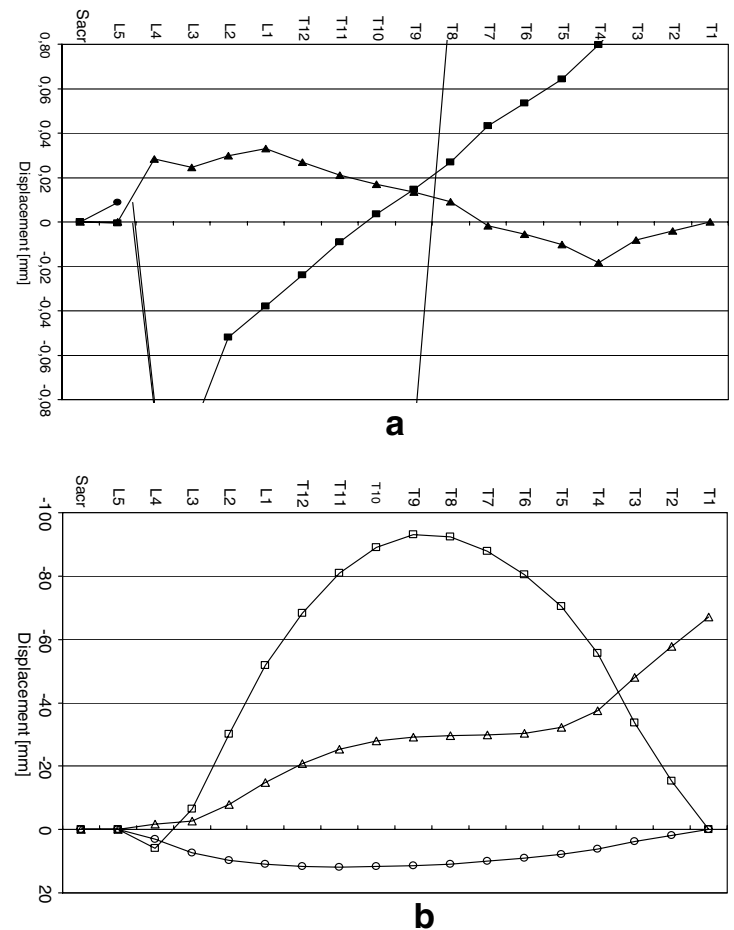

b

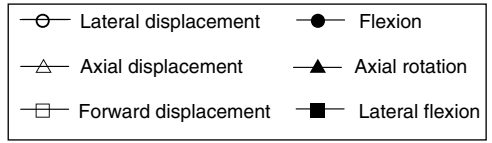

FIGURE 3. Resulting deformations of the spine after buckling (above) and in detail (below). a: rotations; b: displacements. The applied coordinate system is depicted in Fig. 2.

disturbance in the neuromuscular control system and a second stage during adolescent growth in which the scoliotic curve is exacerbated by biomechanical factors. There are two philosophies about the nature of this disturbance: the first one is an anterior-posterior asymmetry as proposed by Somerville ${ }^{31}$ and studied more recently by Dickson. ${ }^{6,7}$ The second one is a leftright asymmetry of muscle properties that disturb the force balance and produce a scoliotic curve. ${ }^{13,32,36}$

It is generally believed that vertebral bodies grow in length by means of plates of epiphysial cartilage on the cephalic and caudal surfaces.

Fusion of the epiphysial growth plates in the limbs stops longitudinal growth of both bones and associated soft tissues. In contrast, progressive distraction causes tension and growth in soft tissues. It seems likely, therefore, that growth at the growth plate causes elongation of the limb, and generating tension in the soft tissues, causes soft tissue growth. By analogy, it is likely that longitudinal growth of the spine is generated by the growth plates of the vertebral bodies and thus growth in the posterior ligaments. Growth plate growth is influenced by the load across the growth plate, so it is likely that the tension in the posterior column will tend to constrain the growth of the vertebral bodies. Under normal circumstances the rate of growth of the anterior column will be in equilibrium with the posterior column. If this equilibrium is lost, and the growth of the anterior column outstrips the growth in the posterior column, then only a small lateral deviation will cause scoliosis, according to the theory of White. ${ }^{37}$

The biomechanical model of the spine, presented in this article to study the initiation of scoliosis, is based on stiffness data of motion segments, described by Panjabi et al. $^{21}$, which are generally considered to be a good basis for a biomechanical description of the vertebral column. A limitation of these stiffness data is the validity for small loads only, but since our study focuses on scoliosis initiation, which is (according to our study) not related with high loads, the data of Panjabi et al. ${ }^{21}$ can be applied. In combination with the orientation of facet joints, the geometry of vertebrae and prestrain of ligaments and muscles, a biomechanical model of the vertebral column has been realized. The ribs and several muscles are not included. The large amount of muscles and the complexity of their orientation makes it very difficult to include 

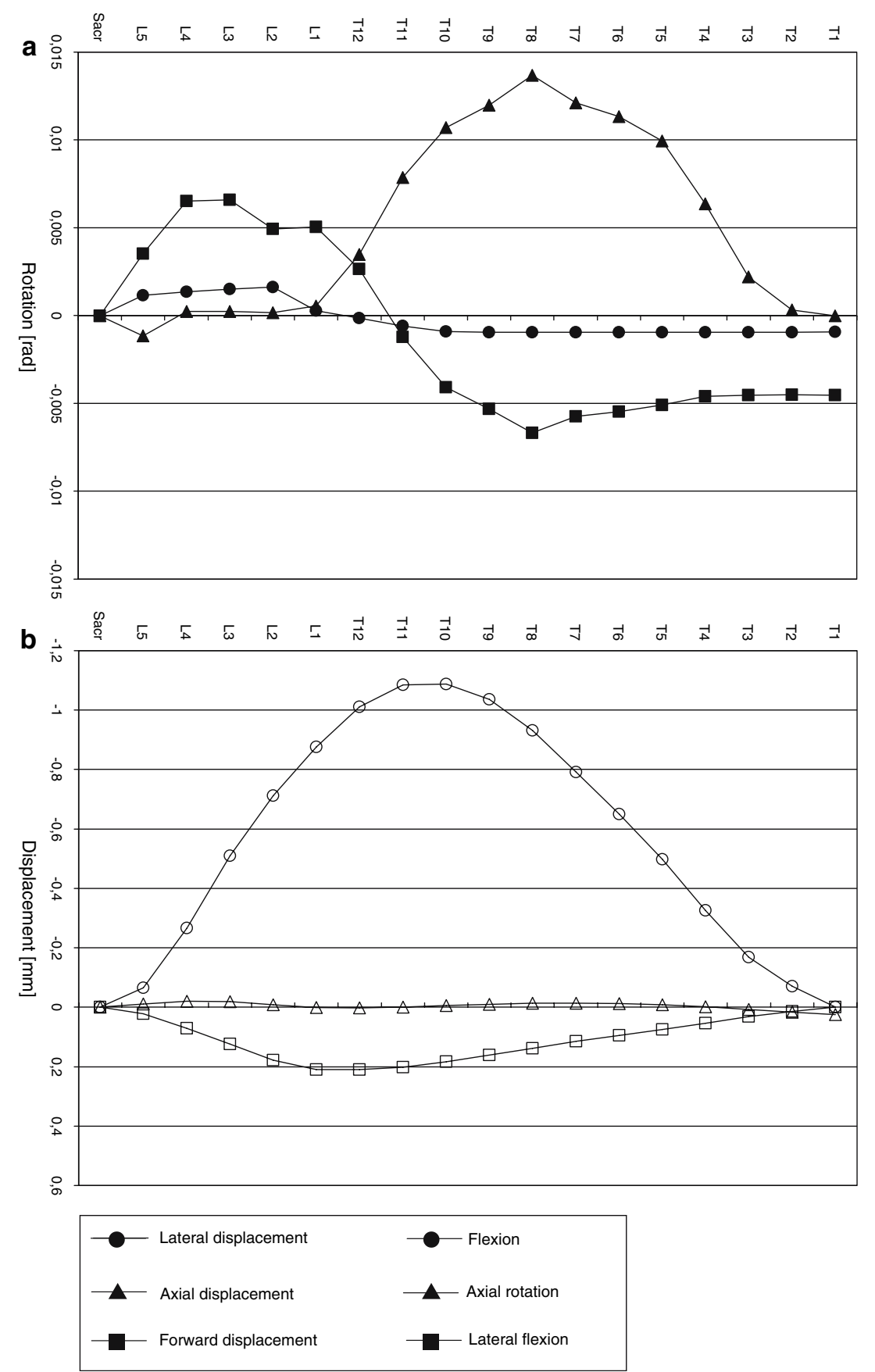

FIGURE 4. Deformations of the spine characterized by an asymmetric stiffness of the MM Rotatores under an axial load of $500 \mathrm{~N}$, relative to the deformation of the symmetrical spine. a: rotations; b: displacements. The applied coordinate system is depicted in Fig. 2.

them. We only included the erector spinae muscles and the muscles of the back (indirectly via the boundary conditions) and the MMR (directly). The influence of the ribs is limited, but not negligible. Improving the model by incorporating ribs and muscles is a challenging task.

The value of $0.5 \%$ prestrain of ligaments and MMR, arbitrarily chosen since no reliable data were available, needed correction. After this correction the deformation data of the finite element model matched well with the deformation data of Panjabi et al. ${ }^{21}$

The maximum calculated ligament stress was found in the facet capsular ligament and appeared to be 2.3 MPa; according to Goel $^{10}$ and to White and Panjabi ${ }^{38}$ this remains below the fatigue strength of the ligaments $(10 \mathrm{MPa})$. 

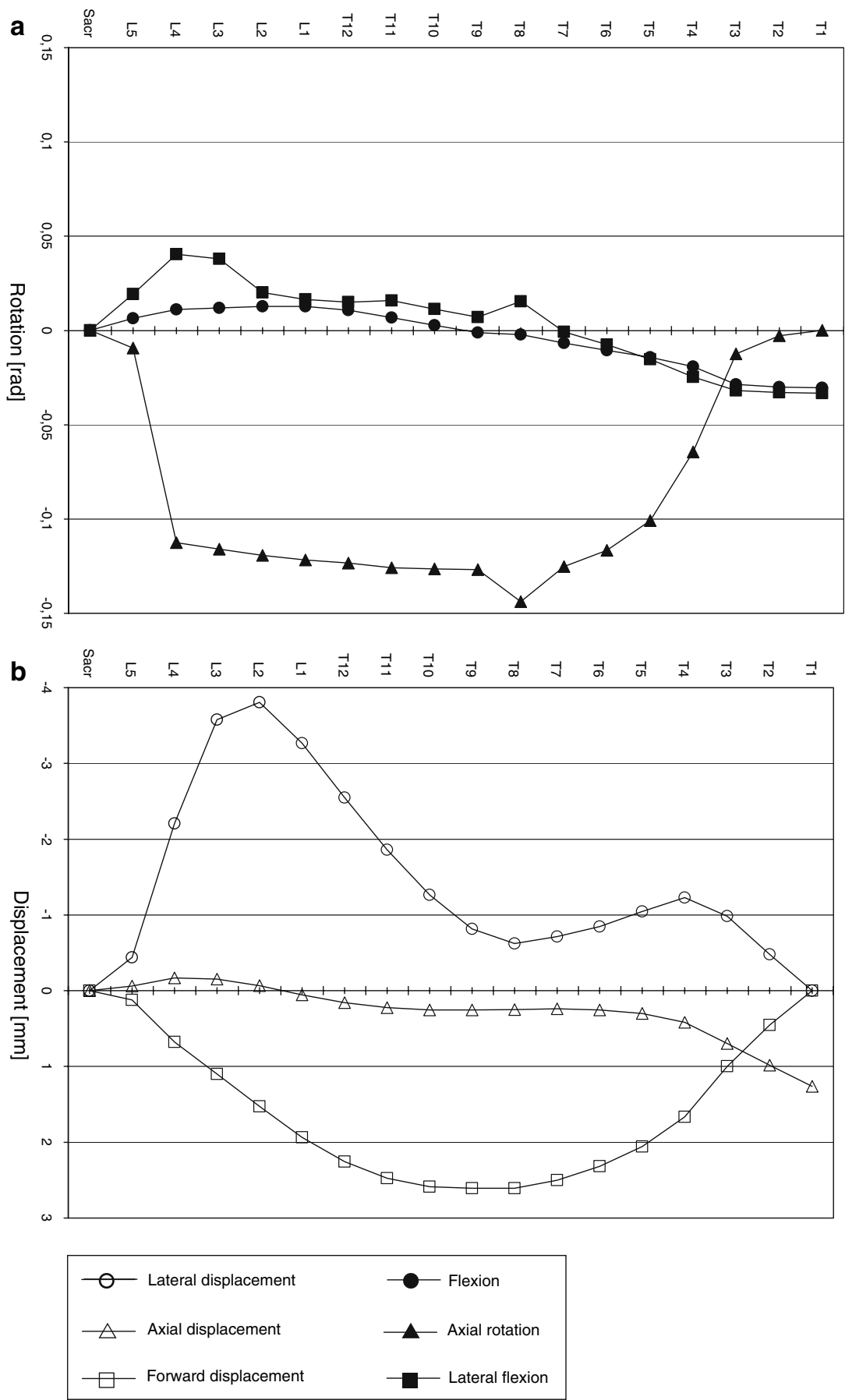

FIGURE 5. Deformations of the spine characterized by an asymmetric stiffness of the intertransverse ligament and ligamentum flavum under an axial load of $500 \mathrm{~N}$, relative to the deformation of the symmetrical spine. a: rotations; b: displacements. The applied coordinate system is depicted in Fig. 2.

During the validation study we noticed that the model was rather sensitive for prestrain values and that prestrain values had a large influence on the mechanical behavior of the spinal column. In our opinion, models that do not include prestrain will show a less realistic mechanical behavior.

The axial buckling load was $250 \mathrm{~N}$, which is comparable to the findings of Lucas and Bresler. ${ }^{16}$ When an axial buckling load was applied, the spinal column becomes shorter, since at $\mathrm{T} 1$ the $\mathrm{y}$-coordinate is $67 \mathrm{~mm}$ lowered. At T1 a distinct flexion of $57^{\circ}$ is found, which is mainly caused by the boundary conditions that leave flexion unconstrained. In reality, C7 will limit this rotation, but for our boundary conditions we only could allow maximal freedom or full constriction. The $\mathrm{S}$-shape of the spine has become more pronounced as 
can be seen by the change in the $z$-coordinate. However, at the point of mechanical instability the deformed spine does not show the characteristics of scoliosis, since only for the lumbar part and the lower thoracic vertebrae lateral deviation is accompanied by an axial rotation of the vertebrae with rotation of the spinous processes towards the concave side. When compared to the results of Goto et al. ${ }^{11}$ who found several buckling modes, our study found only one mode in lateral deviation due to different boundary conditions. The reason that Goto et al. ${ }^{11}$ found a scoliotic curve in contrast to our study could be the incompleteness of their model (no ligaments, articulations and muscles) and thus the absence of prestrain in muscles and ligaments. A second explanation is the type of boundary conditions. The boundary condition of the model of Goto are not described in his article, but from the figures in his article it can be derived that no boundary conditions are present, in contrast to our model that includes more realistic restrictions of horizontal displacements and axial rotations at T1-level, since in real life these displacements and rotations are also restricted by muscle actions.

It has to be mentioned that the spinal deformation studied in this way actually cannot be called buckling, since according to the linear buckling theory of Euler, slender rods are assumed, straight and incompressible, with no or very small imperfections and with linear characteristics. $^{26}$ The human spine, however, is not incompressible, not straight, but inhibits the characteristic S-shape and is composed of different coupled elements with non-linear characteristics. The way we calculated the deformation of the spine under an axial load using the finite element model is correct, since it does not require the assumptions, mentioned above.

Asymmetric stiffness of the MMR results in spinal deformations that are much smaller when compared to deformations of a spinal column with asymmetric stiffness of the intertransverse ligament and the ligamentum flavum. Apparently the influence of MMR on the shape of the spine is less, which is in accordance with the in vivo experience that the spinal ligaments and facet joints mainly determine the passive stiffness of the spinal column. It is thus also imaginable that postponement in growth of the MMR will not result in scoliosis, as was found in this study. The finding that asymmetric stiffness of the ligaments produce a scoliotic curve is in accordance with the results of Stokes and Gardner-Morse (1971), who found an initiation of scoliosis by posterior soft tissue tethering.

Since during the validation study a delicate tuning appeared to be necessary to balance the spinal column, already a small left-right disturbance could destroy this balance and, according to this study, result in scoliosis. It is imaginable that during growth such a small imbalance can occur by a postponement in growth of ligaments. The fact that especially slender girls in their growth spurt are subjected to scoliosis supports this hypothesis, since growth is rapidly and their relatively weak spinal column is extra vulnerable for any disturbance.

\section{CONCLUSIONS}

The developed non-linear 3D finite element model of the human thoracolumbar spine appeared to be valid, since it matches the results of Panjabi et al. ${ }^{21}$

Analyzing the results of the model it can be stated that from the subset of possible mechanisms that were investigated only asymmetric stiffness of the intertransverse ligament and the ligamentum flavum initiate scoliosis, whereas asymmetric stiffness of MMR and buckling do not cause scoliosis.

This finding supports the hypothesis that scoliosis is caused by unilateral postponement in growth of ligaments of the spine and is in contrast with the hypothesis that a defect in the neuromuscular control system initiates scoliosis, at least when it concerns the control of MMR.

\section{REFERENCES}

${ }^{1}$ Alexander, M. A., W. H. Bunch, and S. O. E. Ebbeson. Can experimental dorsal rhizotomy produce scoliosis. $J$. Bone Joint Surg. 54A:1509-1513, 1972.

${ }^{2}$ Andriacchi, T., A. Schultz, T. Belytschko, and J. O. Galante. A model for studies of mechanical interactions between the human spine and rib cage. J. Biomech. 7:497507, 1974.

${ }^{3}$ Beguiristain, J. L., J. de Salis, J. Oriaifo, and J. Canãdell. Experimental scoliosis by epiphysiodesis in pigs. Int. Orthopaed. 3:317-321, 1980.

${ }^{4}$ Cheung, J., A. G. Veldhuizen, W. J. Sluiter, and J. R. van Horn. Geometric and electromyographic assessments in the evaluation of curve progression in idiopathic scoliosis. Spine 31(3):322-329, 2006.

${ }^{5}$ Descrimes, J. L., C. E. Aubin, and F. Boudrealt. Modellling of facet joints in a global finite element model of the spine: Mechanical aspects. Proceedings of the second international symposium on 3-D scoliotic deformities. Pescara, 1994.

${ }^{6}$ Dickson, R. A. The etiology and pathogenesis of idiopathic scoliosis. Acta Orthop. Belg. 58(Suppl 1):21-25, 1992a.

${ }^{7}$ Dickson, R. A. The scientific basis of treatment of idiopathic thoracic scoliosis. Acta Orthop. Belg. 58(Suppl 1):107-110, 1992b

${ }^{8}$ Evans, F. G. Bibliography on the physical properties of the skeletal system. Artif. Limbs 11:48-66, 1967.

${ }^{9}$ Gilbertson, L. G., V. K. Goel, and A. G. Patwardhan. Biomechanics of spinal hyperextension orthoses used in the treatment of thoracolumbar injuries. International Society 
for the Study of the Lumbar Spine, Seattle WA, USA, 1994.

${ }^{10}$ Goel, V. K., W. Kong, J. S. Han, J. N. Weinstein, and L. G. Gilbertson. A combined finite element and optimization investigation of the lumbar spine mechanics with and without muscles. Spine 18:1531-1541, 1993.

${ }^{11}$ Goto, M., N. Kawakami, H. Azegami, Y. Matsuyama, K. Takeuchi, and R. Sasaoka. Buckling and bone remodeling as factors in the development of idiopathic scoliosis. Spine 28:364-370, 2003.

${ }^{12}$ Gross, C., J. Graham, M. Neuwirth, and J. Pugh. Scoliosis and growth, an analysis of the literature. Clin. Orthop. Rel. Res. 175:243-250, 1983.

${ }^{13}$ Herman, R., J. Mixon, A. Fisher, R. Maulucci, and J. Stuyck. Idiopathic scoliosis and the central nervous system: a motor control problem. Spine 10:1-14, 1985.

${ }^{14}$ Langenskiöld, A., and J. E. Michelsson. Experimental progressive scoliosis in the rabbit. J. Bone Joint Surg. 43B:116-120, 1961.

${ }^{15}$ Lowe, T. G., M. Edgar, J. Y. Margulies, N. H. Miller, V. J. Raso, K. A. Reinker, and C.-H. Rivard. Etiology of idiopathic scoliosis: current trends in research. J. Bone Joint Surg. 82-A:1157-1168, 2000

${ }^{16}$ Lucas, D. B., and B. Bresler. Stability of the ligamentous spine. Technical report 40, UCLA San Francisco, Biomechanics Lab, 1961.

${ }^{17}$ Markolf, K. L. Deformation of the thoracolumbar intervertebral joints in response to external loads. J. Bone Joint Surg. 54-A:511-533, 1972.

${ }^{18}$ Miller, N. H. Cause and natural history of adolescent idiopathic scoliosis. Orthopedic Clin. North America 30:343-352, 1999.

${ }^{19}$ Millner, P. A., and R. A. Dickson. Idiopathic scoliosis: biomechanics and biology. Eur. Spine J. 5:362-373, 1996.

${ }^{20}$ Nachemson, A. L., and J. H. Evans. Some mechanical properties of the third human lumbar interlaminair ligament (ligamentum flavum). J. Biomech. 1:211-220, 1968.

${ }^{21}$ Panjabi, M. M., R. A. Brand, and A. A. White III. Threedimensional flexibility and stiffness properties of the human thoracic spine. J. Biomech. 9:185-192, 1976.

${ }^{22}$ Pitzen, T., F. H. Geisler, D. Matthis, H. Müller-Storz, K. Pederson, and W.-I. Steudel. The influence of cancellous bone density on load sharing in human lumbar spine: a comparison between an intact and a surgically altered motion segment. Eur. Spine J. 10:23-29, 2001.
${ }^{23}$ Poelstra, K. A., M. F. Eijkelkamp, and A. G. Veldhuizen. The geometry of the human paraspinal muscles with the aid of three-dimensional computed tomography scans and 3Space Isotrak. Spine 25:2176-2179, 2000.

${ }^{24}$ Roberts, S. B., and P. H. Chen. Elastostatic analyses of the human thoracic skeleton. J. Biomech. 3:527-545, 1970.

${ }^{25}$ Robin, G. C., and H. Stein. Experimental scoliosis in primates. J. Bone Joint Surg. 57B:142-145, 1975.

${ }^{26}$ Scholten, P. J. Idiopathic Scoliosis, PhD Thesis, Free University of Amsterdam. Free University Press, 1986.

${ }^{27}$ Schultz, A. B., D. R. Benson, and C. Hirsch. Forcedeformation properties of human costo-sternal and costovertebral articulations. J. Biomech. 7:311-318, 1974.

${ }^{28}$ Scoles, P. V., A. E. Linton, B. Latimer, M. E. Levy, and B. F. Digiovani. Vertebral body and posterior element morphology: the normal spine in middle life. Spine 10:10821086, 1988

${ }^{29}$ Sevastikoglou, J. A., S. Aaro, T. S. Lindholm, and M. Dahlborn. Experimental scoliosis in growing rabbits by operations on the rib cage. Clin. Orthop. 136:282-286, 1978 .

${ }^{30}$ Smith, R. M., and R. A. Dickson. Experimental structural scoliosis. J. Bone Joint Surg. 69-B:576-581, 1987.

${ }^{31}$ Somerville, E. W. Rotational lordosis; the development of single curve. J. Bone Joint Surg. Br. 34:421-427, 1952.

${ }^{32}$ Stokes, I. A., and M. Gardner-Morse. Analysis of the interaction between vertebral lateral deviation and axial rotation in scoliosis. J. Biomech. 24(8):753-759, 1991.

${ }^{33}$ Stokes, I. A. F., and J. P. Liable. Three-dimensional osseoligamentous model of the thorax representing initiation of scoliosis by asymetric growth. J. Biomech. 23:589-595, 1990.

${ }^{34}$ Subbaraj, K., D. N. Ghista, and G. R. Viviani. Presurgical finite element simulation of scoliosis correction. J. Biomed. Eng. 11:9-18, 1989.

${ }^{35}$ Tkaczuk, H. Tensile properties of human longitudinal ligaments. Acta Orthop. Scan. Suppl. 115:3-69, 1968.

${ }^{36}$ Veldhuizen, A. G., D. J. Weber, and P. J. Webb. The aetiology of idiopatic scoliosis: biomechanical and neuromuscular factors. Eur. Spine J. 9:178-184, 2000.

${ }^{37}$ White, A. A. Kinematics of the normal spine as related to scoliosis. J. Biomech. 4:405-411, 1971.

${ }^{38}$ White, A. A. III, and M M. Panjabi. Clinical Biomechanics of the Spine. Philadelphia/Toronto: J.B. Lippincott Company, 1990. 